\title{
ADAPTIVE INTEGRATION OVER A TRIANGULATED REGION
}

\section{Elise de Doncker David Kahaner Bruce Starkenburg}

U.S. DEPARTMENT OF COMMERCE National Institute of Standards and Technology

Center for Computing and Applled Mathematlcs

Applled and Computational Mathematics Division

Galthersburg, MD 20899

U.S. DEPARTMENT OF COMMERCE Robert A. Mosbacher, Secretary NATIONAL INSTITUTE OF STANDARDS AND TECHNOLOGY

John W. Lyons, Director 

ADAPTIVE

INTEGRATION OVER

A TRIANGULATED

REGION

\section{Elise de Doncker David Kahaner Bruce Starkenburg}

\author{
U.S. DEPARTMENT OF COMMERCE \\ Natlonal Institute of Standards \\ and Tochnology \\ Center for Computing and Applled \\ Mathematles \\ Applled and Computatlonal Mathematles \\ Divislon \\ Galthersburg, MD 20899
}

August 1990

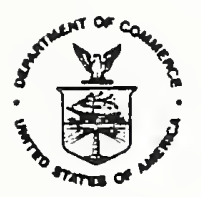

U.S. DEPARTMENT OF COMMERCE Robert A. Mosbacher, Secretary NATIONAL INSTITUTE OF STANDARDS AND TECHNOLOGY

John W. Lyons, Director 



\title{
Adaptive Integration over a Triangulated Region \\ Elise de Doncker, ${ }^{1}$ California Institute of Technology \\ David K. Kahaner and Bruce Starkenburg \\ National Institute of Standards and Technology
}

\begin{abstract}
Authors' Addresses
Elise de Doncker, California Institute of Technology, Mail Stop 158-79, Pasadena, CA 91125.

David K. Kahaner, National Institute of Standards and Technology, Applied and Computational Mathematics Division, Room A151 Technology Building, Gaithersburg, MD 20899.

C. Bruce Starkenburg, National Institute of Standards and Technology, Scientific Computing Environments Division, Room B146 Technology Building, Gaithersburg, MD 20899.
\end{abstract}

Abstract: We present an adaptive algorithm which can be used for integration over a triangulated two-dimensional region $D$. The integrand function may depict some types of singularity on subdivision lines. The algorithm produces a sequence of approximations to the integral over $D$ such that an extrapolation to its limit can be applied. The algorithm is a generalization of the TRIEX algorithm by de Doncker and Robinson.

Categories and subject descriptors: G.1.4 Numerical Analysis: Quadrature and Numerical Differentiation - adaptive quadrature, multiple quadrature; G.4 Mathematics of Computing: Mathematical Software - algorithm analysis, certification and testing; G.m Mathematics of Computing: Miscellaneous - Fortran General Terms, Theory: Algorithms

Additional Key Words and Phrases: Automatic quadrature, numerical multidimensional integration, epsilon-algorithm, singular integrands

\section{Introduction}

The TRIEX program $[1,2]$ was developed for the automatic adaptive quadrature of a user-supplied function over a triangle. The method used in TRIEX is suited to functions with some types of singularity on the edges of the given triangle or on subdivision lines of the adaptive partitioning strategy. In case of a local difficult behaviour of the integrand within the triangle, one can proceed by splitting the original triangle up so that the problem occurs on boundaries of the subtriangles and call TRIEX on each of the subtriangles successively.

However, setting the accuracy requirements on the separate triangles is hard if a relative error has to be satisfied on the entire region. Imposing a too restrictive tolerance on triangles which have a small contribution to the overall

\footnotetext{
${ }^{1}$ On leave from Western Michigan University, Department of Computer Science, Kalamazoo, MI 49008-5021
} 
integral, leads to unnecessary work. Furthermore, if the integral over the entire region is small while the contributions over some triangles are large in absolute value, it may be troublesome to perform the separate integrations to sufficient accuracy.

We have extended and modified the TRIEX method so that it will deal with a triangularized region, by invoking the subdivision process on the set of triangles. The resulting Fortran subroutine is named TRISET in single and DTRIST in double precision. The strategy is similar to that of the quadrature routine (D)QAGP in Quadpack [17], which integrates over an interval that is split up at interior points where some integrand difficulties may occur. As such, the TRISET algorithm also copes with various local singularities on edges of the given triangles.

In the present method, the extrapolation (by the $\epsilon$-algorithm [19]) is carried out on a sequence of results each of which is an approximation to the integral over the entire set of triangles and corresponds to a specific level of subdivision of this set. Each element in the sequence is obtained in a global adaptive way. The algorithm and its theoretical background are outlined in the next section, the structure and use of the program in section 3 ; some examples will be given in section 4 .

\section{Algorithm}

Let

$$
D=\bigcup_{k=1}^{N} \Delta_{k},
$$

where the $\Delta_{k}$ are given non-overlapping triangles (by this we mean that the intersections of their interiors are pairwise disjoint). Then an approximation $Q_{D}(f)$

$$
Q_{D}(f) \approx I_{D}(f)=\int_{D} f(x, y) d x d y
$$

is required, satisfying

$$
\left|I_{D}(f)-Q_{D}(f)\right| \leq \max \left(t_{a}, t_{\mathbf{r}}\left|I_{D}(f)\right|\right)
$$

where $t_{a}$ and $t_{r}$ are user-prescribed absolute and relative tolerances respectively.

Given a symmetric basic rule $Q$ for the triangle, we denote

$$
Q_{D}^{(m)}(f) \equiv \sum_{k=1}^{N} Q_{\Delta_{k}}^{(m)}(f),
$$

where

$$
Q_{\Delta_{k}}^{(m)}(f), \quad \text { for } \quad k=1, \ldots, N
$$

represents the $m^{2}$-copy of $Q$ on $\Delta_{k}$, obtained by a scaled application of the basic rule to the subtriangles in the $m^{2}$-subdivision of $\Delta_{k}[11,12,13,16]$. Thus each 
summand is a sum of integration results over a partition of $\Delta_{k}$ with subtriangles of size $m^{-2}$ times the area of $\Delta_{k}$.

If each of the quadrature rule sums $Q_{\Delta_{k}}^{(m)}(f)$ has an error functional expansion which allows an extrapolation by the $\epsilon$-algorithm on the sequence

$$
\left\{Q_{\Delta_{k}}^{\left(2^{i}\right)}(f)\right\}, \quad i=0,1, \ldots,
$$

then the $\epsilon$-algorithm can also be applied to the sum expansion related to

$$
\left\{Q_{D}^{\left(2^{2}\right)}(f)\right\}, \quad i=0,1, \ldots
$$

Note that terms of the same nature (dependence on $m$ ) in the error expansions arising from different $\Delta_{k}$, will be eliminated from the sum expansion at the same time. If, however, the global expansion is complicated, many extrapolations may be needed to obtain the requested accuracy.

A typical error expansion which allows the use of the $\epsilon$-algorithm for extrapolation [4] is one with terms in

$$
\left(\log ^{\nu_{j}} m\right) / m^{\alpha_{j}}, \quad \nu_{j} \geq 0 \text { integer, and } \alpha_{j}>0 \text { real, }
$$

if one extrapolates on a sequence with a geometric progression in $m$, such as

$$
m=m_{i}=2^{i} \quad \text { for } \quad i=0,1, \ldots
$$

The asymptotic expansions established for integration over the simplex by Lyness $[11,12,13]$ and by Lyness and Monegato [16] are of this type. These include two-dimensional cases where $f(x, y)$ is well-behaved over the triangle apart from possible singularities at the vertices, for example

$$
f(x, y)=r^{\rho} \Theta(\theta) h(\boldsymbol{r}) g(\boldsymbol{x}, y)
$$

over the unit triangle, with

$$
r=\left(x^{2}+y^{2}\right)^{1 / 2}, \quad \theta=\tan ^{-1}(x / y),
$$

and analytic functions $\Theta, h$ and $g$.

Integrands with vertex and edge singularities of the types studied in [14] are also allowed. The latter papers deal with singularities of the form $x^{\lambda} y^{\mu} r^{\rho}$ for integration over the unit square (and the corresponding expansions for the triangle can be derived from it). Edge singularities of the form $x^{\lambda}$ and $x^{\lambda} \log x$ are handled in [18].

The essence of the TRISET algorithm is to replace $Q_{\Delta_{k}}^{\left(2^{i}\right)}(f)$ with a quantity that is computed as a sum over fewer triangles, not all of the same area. Thus TRISET will approximate each $Q_{D}^{\left(2^{i}\right)}(f), i=0,1, \ldots$, by a quantity

$$
A_{i}(f)=\sum_{k=1}^{N} A_{\Delta_{k}}^{\left(2^{i}\right)}(f) \approx Q_{D}^{\left(2^{i}\right)}(f)
$$


where each summand is a sum of integration results over a partition of $\Delta_{k}$ with subtriangles of sizes $\geq 2^{-2 i} \cdot \operatorname{area}\left(\Delta_{k}\right)$ as opposed to exactly $2^{-2 i} \cdot \operatorname{area}\left(\Delta_{k}\right)$ in the full $2^{2 i}$-subdivision of $\Delta_{k}$.

Let us refer to the calculation of $A_{i}(f)$ as "stage $i$ " of the algorithm. Stage 0 simply involves the application of $Q$ to the given triangles. At the start of each further stage $(i>0)$, all triangles in the partition of $D$ are "active", meaning that a subtriangle belonging to the partition of $\Delta_{k}$ is of size $>2^{-2 i} \cdot \operatorname{area}\left(\Delta_{k}\right)$ and hence can be selected for subdivision in the course of this stage.

A stage (except for stage 0 ) is built up as a succession of subdivision steps. A subdivision step comprises the selection of the triangle with the largest error estimate from the active set, subdividing it into 4 similar triangles, computing estimates for the integrals and errors over the new subtriangles, checking the termination criteria and (if not terminated) updating the list of triangles. At the end of the $i$-th stage, $Q_{D}^{\left(2^{2}\right)}(f)$ has been successfully approximated, i.e. the sum of the error estimates over the active set has become small enough, and $A_{i}(f)$ is used as a new extrapolation entry.

The algorithm can be represented as given in Figure 1. Details involving the termination conditions, computation of the local integral and error estimates and the extrapolation are as in the TRIEX algorithm.

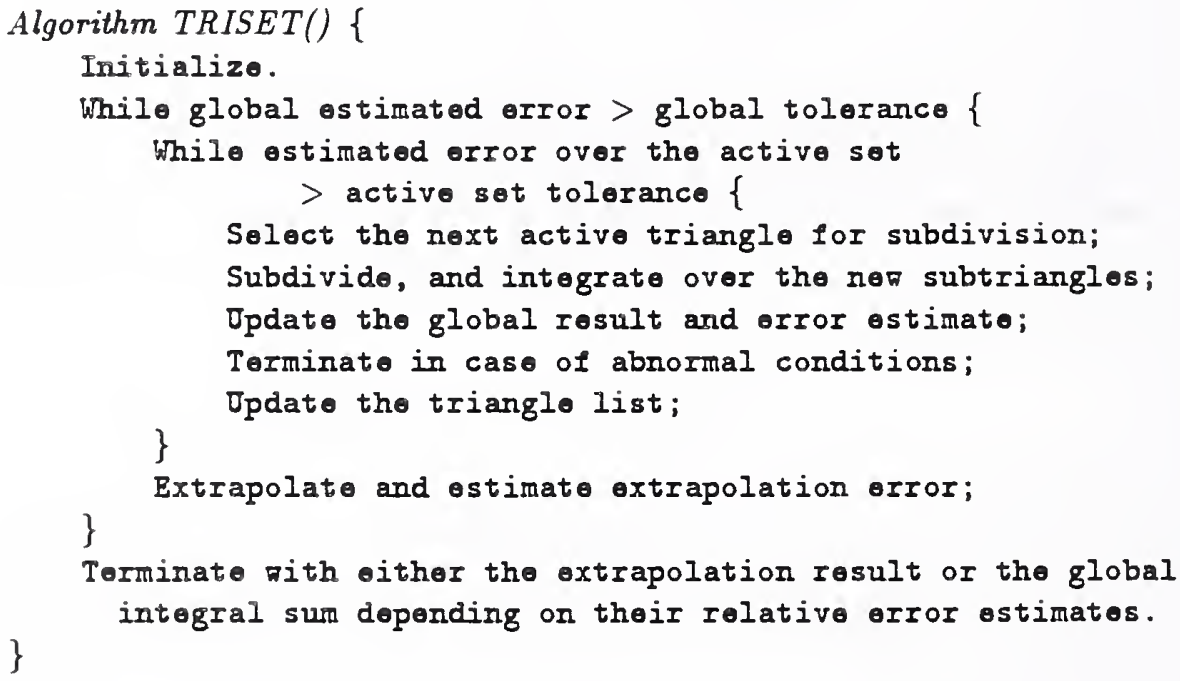

Figure 1: Algorithm description 
3. Use and structure of the program

The integrator is written in Fortran, and is called as

CALL TRISET ( $F, N, X, Y$, EPSABS , EPSREL , MEVALS, ICLOSE , MAXTRI,

* RESULT , ABSERR, NEV ALS , NTR, IER, WRK, IWRK)

TRISET returns an approximation RESULT to the sum $I$ of the integrals of the function $\mathrm{F}(\mathrm{X}, \mathrm{Y})$ over the given set of triangles, and tries to satisfy an accuracy requirement of the form

$$
|I-R E S U L T| \leq \max \{\operatorname{EPSABS}, \operatorname{EPSREL} \cdot|I|\}
$$

where EPSABS and EPSREL are the requested absolute and relative accuracies. The user specifies $\mathrm{N}$ triangles by supplying the abscissas and ordinates of their vertices in $\mathrm{X}\left(3,{ }^{*}\right)$ and $\mathrm{Y}\left(3,{ }^{*}\right)$, respectively. MEVALS is a user-set limit on the number of $\mathrm{F}(\mathrm{X}, \mathrm{Y})$ evaluations. The integrator returns an estimate (often pessimistic) of the absolute error $|I-R E S U L T|$ in ABSERR. If all goes well,

$|I-R E S U L T| \leq A B S E R R \leq \max \{E P S A B S, E P S R E L \cdot|I|\}$.

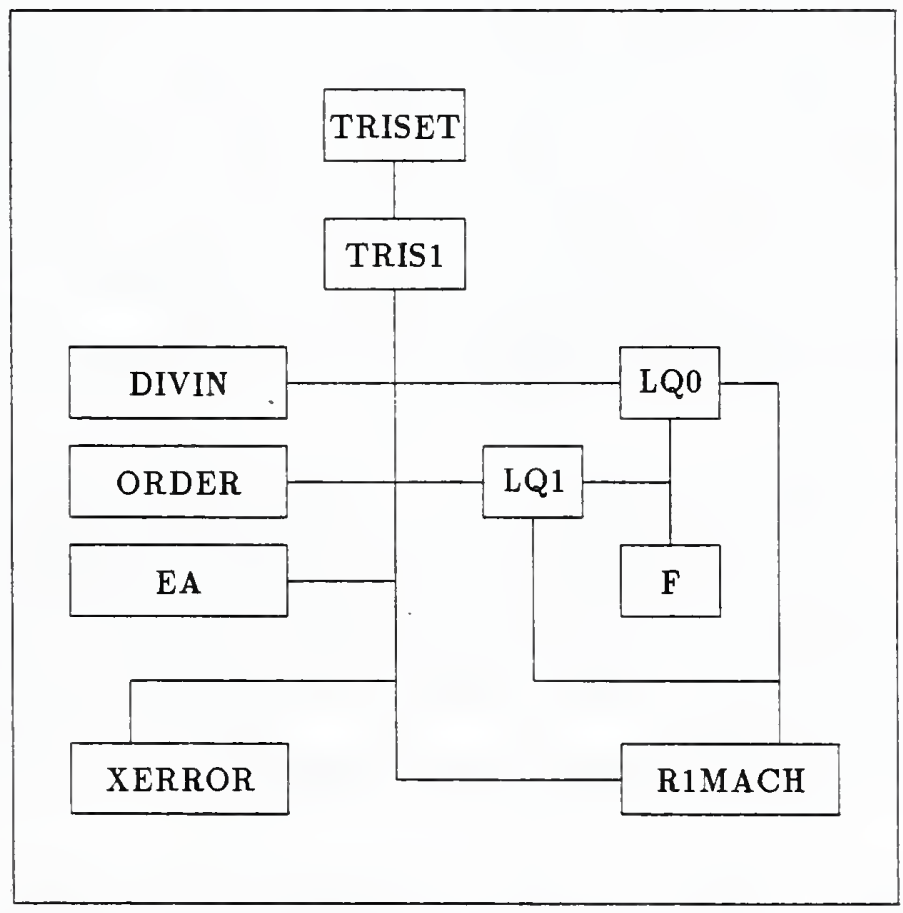

Figure 2: Structure of TRISET 
NEVALS is the number of function evaluations used. When problem situations are detected, an error code is issued in IER.

Two local quadrature modules are provided with the subroutine. The user selects module LQ0 by setting the input parameter ICLOSE to 0. LQ1 is selected by setting ICLOSE to 1 or 2. Each local quadrature module is based on Lyness and Jespersen quadrature rules for the triangle [15]. LQ1 uses the Lyness and Jespersen rule of degree 9 with 19 points and the rule of degree 11 with 28 points. LQ0 uses the Lyness and Jespersen rule of degree 6 with 12 points and the rule of degree 8 with 16 points. LQ1 is usually more accurate than LQ0 for fairly well-behaved functions. However, LQ0, unlike LQ1, uses function values only at interior points of the triangle, so $L Q 0$ can be used in cases where the integrand has singularities on an edge without explicitly defining $F(X, Y)$ there. When a closed rule is used in this case, it is common practice to set the function to 0 where the problem occurs. In some cases of vertex singularities, the related asymptotic error expansions are known to be of the same form as the ones obtained with an open rule [10].

With ICLOSE = 1, the extrapolation is performed as in TRIEX, on the basis of the error expansion for the integral contributions of degree 9 . This alleviates problems with possible corruption of the extrapolation entries in case of singularities on edges, where degree-11 rule evaluations are needed. Note that the degree-11 rule does not require evaluations at the vertices. It can be observed that use of the degree-11 quadrature sums for the extrapolation is often more efficient than the degree- 9 rule in the presence of, for example, a vertex singularity or some types of oscillatory behaviour of the integrand, as is explained by the higher degree of the rule. ICLOSE $=2$ provides the option of extrapolating on the sequence of global quadrature sums obtained with the degree-11 rule. At edge singularities (of the original triangles or subtriangles) it is assumed that the integrand is set to zero by the user.

The open rule used when ICLOSE $=0$, with less points per rule evaluation, is in general efficient when many subdivisions are required to construct each extrapolation entry.

Refer to Figure 2 for a diagram of the routines called by TRISET. Module TRISET allots array storage space and calls TRIS1 which performs the work. R1MACH is the Bell Labs [3] routine which supplies the machine constants needed. XERROR [7] is used for error handling. TRISET is structured similarly to TRIEX, with the main differences being the addition in TRISET of TRIS1 and of two local quadrature modules (TRIEX uses only one closed rule corresponding to LQ1).

Further information involving the usage of the program is given in the Prologue of the routine.

\section{Examples}

In Tables 2 and 3 we show the results from DTRIST for the integration of the functions $\exp (-x-y)\left((1-x)^{2}+y^{2}\right)^{-0 . \theta}$ and $\sqrt{|y(0.5-x)|}(0.5-x)$ respec- 
tively, over the star-shaped region composed by the triangles listed in Table 1 and shown in Figure 3.

\begin{tabular}{|c|l|}
\hline Triangle & Vertices \\
\hline 1 & $(-1 / 3,0)(1,0)(0, .5)$ \\
2 & $(0, .5)(1,0)(.7, .8)$ \\
3 & $(.7, .8)(1,0)(3 \pi, 1)$ \\
4 & $(0, .5)(.7, .8)(.6,22)$ \\
5 & $(-e, 1.7)(-1 / 3,0)(0, .5)$ \\
6 & $(-10,-10)(1 / 3,-.5)(-1 / 3,0)$ \\
7 & $(1 / 3,-.5)(1,0)(-1 / 3,0)$ \\
8 & $(1 / 3,-.5)(1.2,-14)(1,0)$ \\
\hline
\end{tabular}

Table 1: Example Integration Region $D$

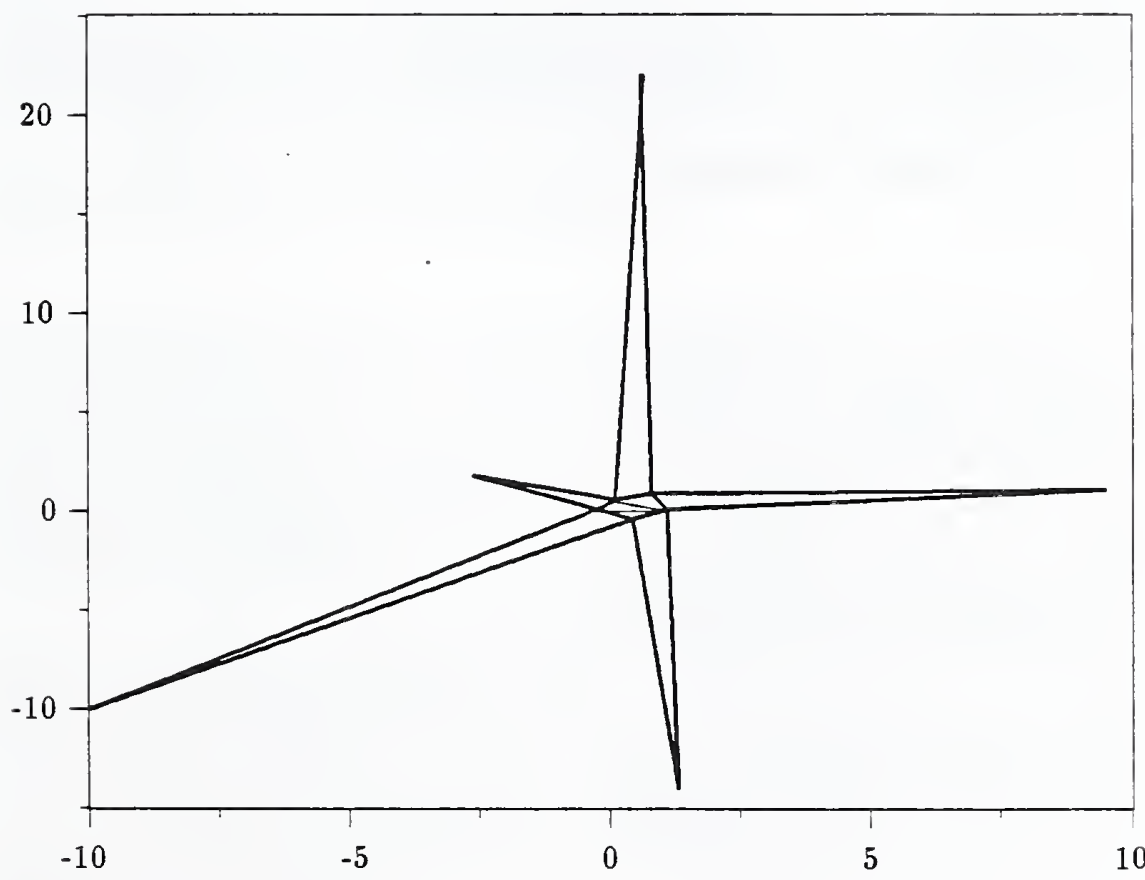

Figure 3: Example Integration Region $D$

For comparison we include the results from TWODQ, a single precision routine that can also be called with a union of triangles [6]. Also included are the results from TRIEX called over the eight triangles successively. TWODQ 


\begin{tabular}{|c|c|c|c|c|c|}
\hline & $\begin{array}{l}\text { Tri- } \\
\text { angle }\end{array}$ & $\begin{array}{l}\text { Integral } \\
\text { approx. }\end{array}$ & $\begin{array}{c}\text { Est. abs. } \\
\text { error }\end{array}$ & $\begin{array}{l}\text { No. of } \\
\text { f-evals. }\end{array}$ & $\begin{array}{c}\text { Act. abs. } \\
\text { error }\end{array}$ \\
\hline \multirow{9}{*}{ TRIEX } & 1 & $0.1060137 \mathrm{D}+01$ & $0.34 \mathrm{D}-07$ & 1292 & \\
\hline & 2 & $0.1351354 \mathrm{D}+01$ & $0.52 \mathrm{D}-07$ & 1292 & \\
\hline & 3 & $0.2846715 \mathrm{D}+01$ & $0.45 \mathrm{D}-06$ & 20872 & \\
\hline & 4 & $0.1322901 \mathrm{D}+00$ & $0.10 \mathrm{D}-06$ & 4496 & \\
\hline & 5 & $0.3393981 \mathrm{D}+00$ & $0.17 \mathrm{D}-07$ & 224 & \\
\hline & 6 & $0.1351640 \mathrm{D}+06$ & $0.81 \mathrm{D}-01$ & 402 & \\
\hline & 7 & $0.1541128 \mathrm{D}+01$ & $0.48 \mathrm{D}-06$ & 1826 & \\
\hline & 8 & $0.2427416 \mathrm{D}+03$ & $0.23 \mathrm{D}-03$ & 10548 & \\
\hline & Total & $0.1354140 \mathrm{D}+06$ & $0.82 \mathrm{D}-01$ & 40952 & $0.13 \mathrm{D}-03$ \\
\hline TWODQ & & & & & \\
\hline ICLOSE $=0$ & & $0.1354139 \mathrm{E}+06$ & $0.13 \mathrm{E}+00$ & 40432 & $0.13 \mathrm{E}+00$ \\
\hline ICLOSE $=1$ & & $0.1354139 E+06$ & $0.14 \mathrm{E}+00$ & 64954 & $0.12 \mathrm{E}+00$ \\
\hline DTRIST & & & & & \\
\hline ICLOSE $=0$ & & $0.1354140 \mathrm{D}+06$ & $0.10 \mathrm{D}-00$ & 11872 & $0.17 \mathrm{D}-01$ \\
\hline ICLOSE $=1$ & & $0.1354140 \mathrm{D}+06$ & $0.23 \mathrm{D}-01$ & 9568 & $0.11 \mathrm{D}-01$ \\
\hline ICLOSE $=2$ & & $0.1354141 \mathrm{D}+06$ & $0.91 \mathrm{D}-01$ & 8280 & $0.48 \mathrm{D}-01$ \\
\hline
\end{tabular}

a Requested relative error for each integration was $10^{-6}$.

does not have any extrapolation capabilities, and is not well suited for the in tegration of functions with singularities. A relative error of $10^{-6}$ was requested for each integration.

Note that the triangles $1,2,3,7$ and 8 all have the point $(1,0)$ as a vertex, where the first integrand has a singularity. In a polar coordinate system centered at $(1,0)$, the function has the singular behaviour of $1 / R^{1.8}$, where $R$ represents the radial coordinate in that system.

The triangles 1 and 7 share an edge along $y=0$ and triangles $2,3,5,6$ and 8 have a vertex on $y=0$, where the second function has a derivative singularity. Furthermore, the second function has higher order derivative singularities along $x=0.5$, which is inside the triangles $1,2,4,7$ and 8 . This was included as an example for which the extrapolation is not expected to be effective.

The TWODQ examples were run on a Control Data Cyber 180/855 system operating under NOS/VE (level 1.5.1) at the National Institute of Standards and Technology. This machine carries about 14 decimal digits in single precision. The results from TRIEX and DTRIST were obtained on a Sun 4/260 under Unix 4.1 at Western Michigan University. About 16 decimals are carried in double precision on this system. 


\begin{tabular}{|c|c|c|c|c|c|}
\hline & $\begin{array}{c}\text { Tri- } \\
\text { angle }\end{array}$ & $\begin{array}{l}\text { Integral } \\
\text { approx. }\end{array}$ & $\begin{array}{c}\text { Est. abs. } \\
\text { error }\end{array}$ & $\begin{array}{l}\text { No. of } \\
\text { f-evals. }\end{array}$ & $\begin{array}{c}\text { Act. abs. } \\
\text { error }\end{array}$ \\
\hline \multirow{9}{*}{ TRIEX } & 1 & $0.2672767 \mathrm{D}-01$ & $0.12 \mathrm{D}-07$ & 25322 & \\
\hline & 2 & $-0.4512156 \mathrm{D}-02$ & $0.36 \mathrm{D}-08$ & 8412 & \\
\hline & 3 & $-0.1950725 \mathrm{D}+02$ & $0.12 \mathrm{D}-04$ & 224 & \\
\hline & 4 & $0.5018726 \mathrm{D}+00$ & $0.35 \mathrm{D}-06$ & 6632 & \\
\hline & 5 & $0.1637102 \mathrm{D}+01$ & $0.15 \mathrm{D}-05$ & 402 & \\
\hline & 6 & $0.1122360 \mathrm{D}+03$ & $0.11 \mathrm{D}-03$ & 402 & \\
\hline & 7 & $0.1315411 \mathrm{D}-01$ & $0.33 \mathrm{D}-08$ & 9302 & \\
\hline & 8 & $-0.2547351 \mathrm{D}+01$ & $0.23 \mathrm{D}-05$ & 3784 & \\
\hline & Total & $0.9235573 \mathrm{D}+02$ & $0.12 \mathrm{D}-03$ & 54480 & $0.19 \mathrm{D}-04$ \\
\hline TWODQ $^{a}$ & & & & & \\
\hline ICLOSE $=0$ & & $0.9235634 \mathrm{E}+02$ & $0.83 E-03$ & 11368 & $0.59 \mathrm{E}-03$ \\
\hline ICLOSE $=1$ & & $0.9235640 \mathrm{E}+02$ & $0.12 \mathrm{E}-02$ & 8084 & $0.64 \mathrm{E}-03$ \\
\hline DTRIST & & & & & \\
\hline ICLOSE $=0$ & & $0.9235575 \mathrm{D}+02$ & $0.17 D-04$ & 6944 & $0.42 \mathrm{D}-05$ \\
\hline ICLOSE $=1$ & & $0.9235576 \mathrm{D}+02$ & $0.92 \mathrm{D}-04$ & 9752 & $0.44 \mathrm{D}-05$ \\
\hline ICLOSE $=2$ & & $0.9235575 \mathrm{D}+02$ & $0.88 \mathrm{D}-04$ & 12328 & $0.98 \mathrm{D}-06$ \\
\hline
\end{tabular}

Table 3: Integration ${ }^{b}$ of $|y(0.5-x)|^{1 / 2}(0.5-x)$ on $D$

\footnotetext{
${ }^{a}$ Requested accuracy was not reached because TWODQ detected roundoff error.

${ }^{b}$ Requested relative error for each integration was $10^{-6}$.
}

\section{Concluding remarks}

Automatic integration over triangles has received considerable attention by several authors $[5,9,1,2,6]$. Applications include integration over irregular regions which can be triangularized or where the integral can be approximated by that over a set of triangles.

TRISET is an extension of the TRIEX algorithm for integration over a triangularized region. It allows dealing efficiently with problems where a relative accuracy is requested for the integral over the entire set and also handles some forms of boundary singularity.

Furthermore TRISET lends itself naturally to parallelization using the task partitioning concept [8], as the user-supplied triangle set and its partitions provide an appropriate task pool, thereby retaining the advantages offered by the extrapolation technique. 


\section{References}

1. de Doncker, E. and Robinson, I. An Algorithm for Automatic Integration over a Triangle using Nonlinear Extrapolation. ACM Trans. Math. Softw. 10,1 (1984), 1-16.

2. de Doncker, E. and Robinson, I. Algorithm 612 - TRIEX: Integration over a Triangle Using Nonlinear Extrapolation. ACM Trans. Math. Softw. 10, 1 (1984), 17-22.

3. Fox, F.A., Hall, A.D. and Schryer, N.L. The PORT Mathematical Subroutine Library. Computer Science Tech. Rep. 47, Bell Laboratories, Murray Hill, New Jersey (1977).

4. Genz, A.C. The Approximate Calculation of Multidimensional Integrals Using Extrapolation Methods. Ph.D. Thesis, Univ. of Kent at Canterbury (1975).

5. Haegemans, A. An Algorithm for the Automatic Integration over a Triangle. Computing 19 (1977), 179-187.

6. Kahaner, D.K. and Rechard, O.W. TWODQD an Adaptive Routine for Two-dimensional Integration. J. Comp. Appl. Math., 17, 1\&2 (1984) 215-234.

7. Jones, R.E. and Kahaner, D.K., XERROR, "The SLATEC Error Handling Package", Sandia Report SAND82-0800 UC-32, Sandia National Laboratories, Albuquerque, NM 87185 (1982).

8. Kapenga, J. and de Doncker, E. A Parallelization of Adaptive Task Partitioning Algorithms. Parallel Computing 7 (1988), 211-225.

9. Laurie, D.P. Algorithm 584 - CUBTRI: Automatic Cubature over a Triangle. ACM Trans. Math. Softw. 8, 2 (1982), 210-218.

10. Lyness, J.N. An Error Functional Expansion for N-Dimensional Quadrature with an Integrand Function Singular at a Point. Math. Comp. 30 (1976), 133, 1-23.

11. Lyness, J.N. Quadrature over the Simplex, Part I. A Representation for the Integrand Function. SIAM J. Numer. Anal. 15, 1 (1978), 122-133.

12. Lyness, J.N. Quadrature over the Simplex, Part II. A Representation for the Error Functional. SIAM J. Numer. Anal. 15, 5 (1978), 870-887.

13. Lyness, J.N. QUG2 - Integration over a Triangle. MCS-TM-13, Argonne National Laboratory (1983). 
14. Lyness, J.N. and de Doncker-Kapenga, E. On Quadrature Error Functional Expansions - Part I. Journal of Computational and Applied Mathematics 17 (1987), 131-149. - Part II. Unpublished.

15. Lyness, J. N. and Jespersen, P. Moderate Degree Symmetric Quadrature Rules for the Triangle. J. Inst. Maths. Applics. 15 (1975), 19-32.

16. Lyness, J.N. and Monegato, G. Quadrature Error Functional Expansions for the Simplex when the Integrand has Singularities at Vertices. Math. Comp. 34, 149 (1980), 213-225.

17. Piessens, R., de Doncker-Kapenga, E., Uberhuber, C.W. and Kahaner, D.K. QUADPACK - A Subroutine Package for Automatic Integration. Springer Series in Computational Mathematics 1 (1983), Springer-Verlag, N.Y.

18. Sidi, A. Euler-Maclaurin Expansions for Integrals over Triangles and Squares of Functions having Algebraic/Logarithmic Singularities along an Edge. Journal of Approximation Theory 39 (1983), 39-53.

19. Wynn, P. On a Device for Computing the $\epsilon_{m}\left(S_{n}\right)$ Transformation. Mathematical Tables and Aids to Computing 10 (1956), 91-96. 



\section{BIBLIOGRAPHIC DATA SHEET}

2. PERFORMING ORGAMIZATION REPORT NUMBER

3. PUBUCATION DATE

August 1990

4. TITLE AND SUBtitLE

Adaptive Integration over a Triangulated

Region

5. AUTHOR(S)

Elise de Doncker, David K. Kahaner, and B. Starkenburg

6. PERFORMING ORGANIZATION (IF JOINT OR OTHER THAN MIST, SEE INSTRUCTIONS)

U.S. DEPARTMENT OF COMMERCE

NATIONAL INSTITUTE OF STANDARDS AND TECHNOLOGY

GAITHERSBURG, MD 20899

7. CONTRACT/GRANT NUMBER

8. TYPE OF REPORT AND PERIOD COVERED

9. SPONSORING ORGANIZATION NAME AND COMPLETE ADDRESS (STREET, CITY, STATE, ZIP)

DOCUMENT DESCRIBES A COMPUTER PROGRAM; SF-185, FIPS SOFTWARE SUMMARY, IS ATTACHED.

11. ABSTRACT (A 200-WORD OR LESS FACTUAL SUMMARY OF MOST SIGNIFICANT IMFORMATION. IF DOCUMENT INCLUDES A SIGNIFICANT BIBLOGRAPHY OR LUTERATURE SURVEY, MENTION IT HERE.)

The authors present an adaptive algorithm which can be used for integration over a triangulated two-dimensional region $\mathrm{D}$. The integrand function may depict some types of singularity on subdivision lines. The algorithm produces a sequence of approximations to the integral over $D$ such that an extrapolation to its limit can be applied. The algorithm is a generalization of the TRIEX algorithm by de Doncker and Robinson

12. KEY WORDS (6 TO 12 ENTRIES; ALPHABETICAL ORDER; CAPITALUZ ONLY PROPER NAMES; AND SEPARATE KEY WORDS BY STEMICOLONS) adaptive quadrature; multiple quadrature; automatic quadrature; numerical multidimensional integration; epsilon-algorithm; singular integrands

13. AVAILABIUTY

E

\section{UNUMITED}

FOR OFFICIAL DISTRIBUTION. DO NOT RELEASE TO NATIONAL TECHNICAL INFORMATION SERVICE (NTIS).

ORDER FROM SUPERINTENDENT OF DOCUMENTS, U.S. GOVERNMENT PRINTING OFFICE, WASHINOTON, DC 20402.

ORDER FROM NATIONAL TECHMICAL INFORMATION SERVICE (NTIS), SPRINGFIELD, VA 22161.
14. NUMBER OF PRINTED PAGES 14

15. PRICE

$\mathrm{AO} 2$ 


